\title{
Tracking Advanced Planetary Systems (TAPAS) with HARPS-N $\mathbf{N}^{\star} \star \star$
}

\section{TYC 3667-1280-1: The most massive red giant star hosting a warm Jupiter}

\author{
A. Niedzielski ${ }^{1}$, E. Villaver ${ }^{2}$, G. Nowak ${ }^{3,4,1}$, M. Adamów ${ }^{5,1}$, G. Maciejewski ${ }^{1}$, K. Kowalik ${ }^{6}$, A. Wolszczan ${ }^{7,8}$, \\ B. Deka-Szymankiewicz ${ }^{1}$, and M. Adamczyk ${ }^{1}$ \\ 1 Toruń Centre for Astronomy, Faculty of Physics, Astronomy and Applied Informatics, Nicolaus Copernicus University in Toruń, \\ Grudziadzka 5, 87-100 Toruń, Poland \\ e-mail: Andrzej.Niedzielski@umk.pl \\ 2 Departamento de Física Teórica, Universidad Autónoma de Madrid, Cantoblanco 28049 Madrid, Spain \\ e-mail: Eva.Villaver@uam.es \\ 3 Instituto de Astrofísica de Canarias, 38205 La Laguna, Tenerife, Spain \\ 4 Departamento de Astrofísica, Universidad de La Laguna, 38206 La Laguna, Tenerife, Spain \\ 5 McDonald Observatory and Department of Astronomy, University of Texas at Austin, 2515 Speedway, Stop C1402, Austin, \\ TX 78712-1206, USA \\ ${ }^{6}$ National Center for Supercomputing Applications, University of Illinois, Urbana-Champaign, 1205 W Clark St, MC-257, Urbana, \\ IL 61801, USA \\ 7 Department of Astronomy and Astrophysics, Pennsylvania State University, 525 Davey Laboratory, University Park, PA 16802, \\ USA \\ e-mail: alex@astro.psu.edu \\ 8 Center for Exoplanets and Habitable Worlds, Pennsylvania State University, 525 Davey Laboratory, University Park, PA 16802, \\ USA
}

Received 1 March 2016 / Accepted 17 March 2016

\begin{abstract}
Context. We present the latest result of the TAPAS project that is devoted to intense monitoring of planetary candidates that are identified within the PennState-Torun planet search.

Aims. We aim to detect planetary systems around evolved stars to be able to build sound statistics on the frequency and intrinsic nature of these systems, and to deliver in-depth studies of selected planetary systems with evidence of star-planet interaction processes.

Methods. The paper is based on precise radial velocity measurements: 13 epochs collected over 1920 days with the Hobby-Eberly Telescope and its High-Resolution Spectrograph, and 22 epochs of ultra-precise HARPS-N data collected over 961 days.

Results. We present a warm-Jupiter $\left(T_{\mathrm{eq}}=1350 \mathrm{~K}, m_{2} \sin i=5.4 \pm 0.4 M_{\mathrm{J}}\right)$ companion with an orbital period of 26.468 days in a circular $(e=0.036)$ orbit around a giant evolved $\left(\log g=3.11 \pm 0.09, R=6.26 \pm 0.86 R_{\odot}\right)$ star with $M_{\star}=1.87 \pm 0.17 M_{\odot}$. This is the most massive and oldest star found to be hosting a close-in giant planet. Its proximity to its host $(a=0.21 \mathrm{au})$ means that the planet has a $13.9 \pm 2.0 \%$ probability of transits; this calls for photometric follow-up study.

Conclusions. This massive warm Jupiter with a near circular orbit around an evolved massive star can help set constraints on general migration mechanisms for warm Jupiters and, given its high equilibrium temperature, can help test energy deposition models in hot Jupiters.
\end{abstract}

Key words. planets and satellites: detection - planets and satellites: individual: TYC 3667-1280-1 b - planet-star interactions stars: late-type

\section{Introduction}

The population of hot Jupiters (HJ), Jupiter-mass planets on short-period orbits $(P<10$ days, or within $0.1 \mathrm{au})$, which were unveiled with the finding of 51 Peg b (Mayor \& Queloz 1995),

\footnotetext{
^ Based on observations obtained with the Hobby-Eberly Telescope, which is a joint project of the University of Texas at Austin, the Pennsylvania State University, Stanford University, Ludwig-MaximiliansUniversität München, and Georg-August-Universität Göttingen.

$\star \star$ Based on observations made with the Italian Telescopio Nazionale Galileo (TNG) operated on the island of La Palma by the Fundación Galileo Galilei of the INAF (Istituto Nazionale di Astrofisica) at the Spanish Observatorio del Roque de los Muchachos of the Instituto de Astrofísica de Canarias.
}

was probably one of the least expected discoveries in search for exoplanets. The origin of this rare class of objects, only known to be present around approximately $0.5-1 \%$ of FGK stars (Wright et al. 2012; Howard et al. 2012), generally involves early (e.g., Type II), or late migration scenarios (following planet-planet scattering or secular perturbations from more distant objects) since formation beyond the snow line is required. Recently, however, the in-situ formation of these systems has also been proposed (Boley et al. 2016).

Warm Jupiters (WJ, Fogg \& Nelson 2009), Jupiter-mass planets on 10-100 day orbits, are even more intriguing. WJ have observed eccentricities too low for significant tidal evolution, and while the missing $\mathrm{HJ}$ from the evolved star-planet population can be naturally explained by engulfment by the 
star, the lack so far of detected WJ requires further explanation (see, e.g., Villaver et al. 2014). Recently, Frewen \& Hansen (2016) invoked Kozai-Lidov oscillations to predict an entire removal of WJ planets by the time the star reaches $R>5 R_{\odot}$, while an identical constant eccentricity population survives beyond $40 R_{\odot}$. The authors argued that the WJ migrate through Kozai-Lidov oscillations. Dawson \& Murray-Clay (2013) presented evidence that both smooth disk migration and dynamical interactions may play a role in $\mathrm{WJ}$ formation, with the efficiency depending on the host's metallicity. Dong et al. (2014) showed that high-eccentricity WJ are more likely to have an external Jovian perturber and migrate through dynamical interactions. This finding was recently supported by Huang et al. (2016), who found that different from $\mathrm{HJ}$, half of the WJ have companions. The mutual inclination of orbits in six such WJ-external perturber pairs reported by Dawson \& Chiang (2014) indeed points to Kozai-Lidov oscillations as a mechanism responsible for the inward migration of WJ.

We here present the discovery of a WJ planet $(P=26.468 \mathrm{~d})$ orbiting an evolved star with $R=6.3 R_{\odot}$ on a circular orbit. Clearly, this unique system can help set constraints on the invoked mechanism for WJ formation through the Kozai-Lidov oscillations. Furthermore, its proximity to a very luminous star means that it has the equivalence temperature of a hot Jupiter. If hot-Jupiter inflation works by depositing irradiation into the planet's deep interiors, then planetary radii should increase in response to the increased irradiation of this evolved star, constraining structure theories on the physics of the hot-Jupiter inflation mechanism (Lopez \& Fortney 2016). Moreover, there is a high probability of transit detection of this unique object, which may then serve as a perfect laboratory for planet formation and migration theories.

The paper is organized as follows: in Sect. 2 we present the available observations and discuss the influence of the stellar activity on the radial velocity (RV) variation measurements. Section 3 shows the results of the Keplerian data modeling, in Sect. 4 we discuss the transit possibilities, and in Sect. 5 we discuss the results and present the conclusions.

\section{Observations, radial velocities, and activity}

TYC 3667-1280-1 (2MASS J00513296+5825342) belongs to a sample of about 300 planetary or brown dwarf (BD) candidates identified in the complete sample of over 1000 stars for RV variations with the $9.2 \mathrm{~m}$ Hobby-Eberly Telescope (HET, Ramsey et al. 1998) and its High-Resolution Spectrograph (HRS, Tull 1998) since 2004 within the PennState - Toruń Centre for Astronomy Planet Search (PTPS, Niedzielski et al. 2007, 2016a) selected for a more intense precise RV followup within Tracking Advanced Planetary Systems (TAPAS) with HARPS-N (Niedzielski et al. 2015, 2016b; Adamów et al. 2015). A summary of the available data for TYC $3667-1280-1$ is given in Table 1.

The spectroscopic observations presented here were made with the HET and its HRS in the queue-scheduled mode (Shetrone et al. 2007) and with the $3.58 \mathrm{~m}$ Telescopio Nazionale Galileo (TNG) and its High-Accuracy Radial velocity Planet Searcher in the Northern hemisphere (HARPS-N, Cosentino et al. 2012).

For HET HRS spectra we used a combined gas-cell (Marcy \& Butler 1992; Butler et al. 1996), and cross-correlation (Queloz 1995; Pepe et al. 2002) method for precise RV and spectral line bisector (BS) measurements, respectively. The
Table 1. Summary of the available data on TYC 3667-1280-1.

\begin{tabular}{ll|ll}
\hline \hline Parameter & Value & Parameter & Value \\
\hline$V(\mathrm{mag})$ & $9.86 \pm 0.02^{(1)}$ & $M / M_{\odot}$ & $1.87 \pm 0.17^{(5)}$ \\
$B-V(\mathrm{mag})$ & $1.00 \pm 0.06^{(1)}$ & $\log L / L_{\odot}$ & $1.38 \pm 0.10^{(5)}$ \\
$(B-V)_{0}(\mathrm{mag})$ & $0.84^{(2)}$ & $R / R_{\odot}$ & $6.26 \pm 0.86^{(5)}$ \\
$M_{V}(\mathrm{mag})$ & $0.99^{(2)}$ & $\log$ age $(\mathrm{yr})$ & $9.13 \pm 0.10^{(5)}$ \\
$d(\mathrm{pc})$ & $481 \pm 37^{(6)}$ & $v_{\text {osc }}\left(\mathrm{m} \mathrm{s}^{-1}\right)$ & $3.00_{-0.95}^{+1.41}(7)$ \\
$T_{\text {eff }}(\mathrm{K})$ & $5130 \pm 24^{(2)}$ & $P_{\text {osc }}(\mathrm{d})$ & $0.075_{-0.024}^{+0.032}(7)$ \\
$\log g$ & $3.11 \pm 0.09^{(2)}$ & $P_{\text {rot }}(\mathrm{d})$ & $99 \pm 19^{(7)}$ \\
{$[\mathrm{Fe} / \mathrm{H}]$} & $-0.08 \pm 0.05^{(2)}$ & $v_{\text {rot }} \sin i_{\star}\left(\mathrm{km} \mathrm{s}^{-1}\right)$ & $3.2 \pm 0.4^{(4)}$ \\
$\mathrm{RV}\left(\mathrm{km} \mathrm{s}^{-1}\right)$ & $9.54 \pm 0.06^{(3)}$ & $A(\mathrm{Li})_{\mathrm{NLTE}}$ & $0.80 \pm 0.21^{(4)}$ \\
\hline
\end{tabular}

References. (1) Høg et al. (2000); (2) Zieliński et al. (2012); (3) Nowak (2012); (4) Adamów et al. (2014); (5) Adamczyk et al. (2016); (6) calculated from $M_{V},(7)$ This work.

implementation of this technique to our data is described in Nowak (2012) and Nowak et al. (2013).

HARPS-N radial velocity measurements and their uncertainties as well as BS were obtained with the standard user pipeline, which is based on the weighted CCF method (Fellgett 1955; Griffin 1967; Baranne et al. 1979, 1996; Queloz 1995; Pepe et al. 2002), using the simultaneous Th-Ar calibration mode of the spectrograph and the K5 cross-correlation mask. The RV and BS data for TYC 3667-1280-1 are presented in Tables A.1 and A.2.

There is no correlation between RV and BS in either HET (Pearson's $r=0.07)$ or TNG $(r=-0.19)$ data, and we can assume that the RV signal origin is Dopplerian. Our $\mathrm{H} \alpha$ index (Maciejewski et al. 2013) variations are neither correlated with the observed RVs $(r=-0.17)$ nor with the control line FeI $6593.878 \AA(r=0.37)$ and therefore show no trace of stellar activity. The $\mathrm{Ca}$ II $\mathrm{H}$ and $\mathrm{K}$ lines present in the TNG HARPS-N spectra allow us to calculate the instrumental $S_{H K}^{\text {inst }}$ index (Niedzielski et al. 2015), which is $0.17 \pm 0.08$ and indicates that TYC 3667-1280 is not an active star. There are no emissions in the core of $\mathrm{H}$ and $\mathrm{K}$ lines.

Extensive photometry for TYC 3667-1280-1 is available from NSVS (Woźniak et al. 2004) and WASP (Pollacco et al. 2006). The 157 epochs of NSVS photometry collected between JD 2451336.35-2451566.14 show constant light $m_{\mathrm{NSVS}}=$ $9.66 \pm 0.03 \mathrm{mag}$ and no trace of variability. 1833 epochs of WASP photometry, obtained from the original 2064 through three-sigma iterative filtering were collected between JD 2454327.73-2454452.35 and are partly contemporaneous with our HET HRS data. They show $m_{\text {WASP }}=9.927 \pm$ 0.009 mag, varying within $0.022 \mathrm{mag}$ and a trace of weak periodic signal at 15.67 days. This signal is well separated from that present in RV and also too short to be related to stellar rotation and a possible spot on the stellar surface.

\section{Keplerian analysis}

Keplerian orbital parameters were derived using a hybrid approach (e.g., Goździewski et al. 2003, 2007; Goździewski \& Migaszewski 2006), in which the PIKAIAbased, global genetic algorithm (GA; Charbonneau 1995) was combined with MPFit algorithm (Markwardt 2009) to find the best-fit Keplerian orbit delivered by RVLIN (Wright \& Howard 2009) modified to allow the stellar jitter to be fitted as a free parameter (Ford \& Gregory 2007; Johnson et al. 2011). The 


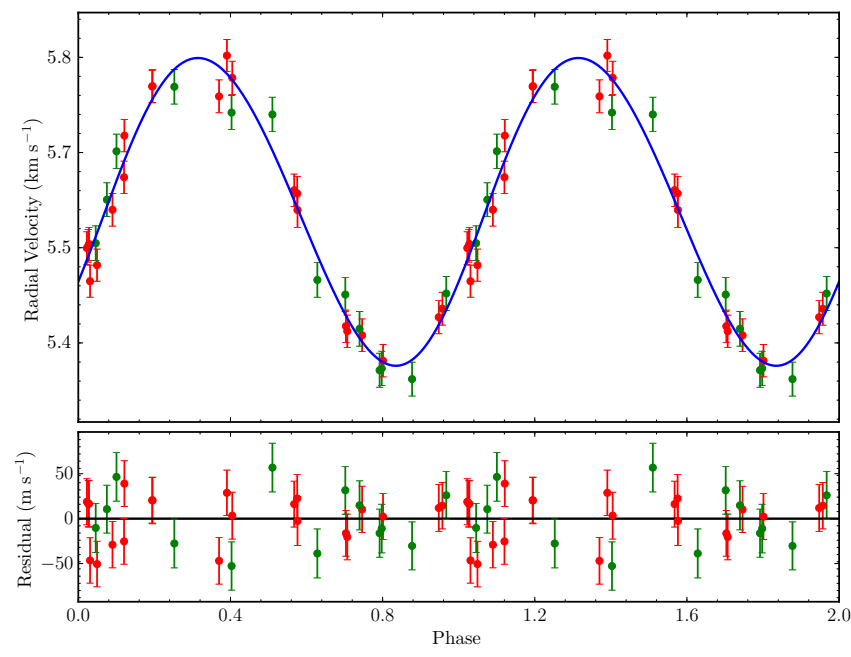

Fig. 1. Keplerian best fit to combined HET HRS (green points) and TNG Harps-N (red points) data for TYC 3667-1280-1. The jitter is added to uncertainties.

Table 2. Keplerian orbital parameters of TYC 3667-1280-1 b.

\begin{tabular}{ll|ll}
\hline \hline Parameter & Value & Parameter & Value \\
\hline$P($ days $)$ & $26.468_{-0.005}^{+0.005}$ & $v_{0}\left(\mathrm{~m} \mathrm{~s}^{-1}\right)$ & $5610.4_{-2.2}^{+2}$ \\
$T_{0}(\mathrm{MJD})$ & $56319.6_{-3.3}^{+3.7}$ & offset $\left(\mathrm{m} \mathrm{s}^{-1}\right)$ & $5545_{-11}^{+11}$ \\
$K\left(\mathrm{~m} \mathrm{~s}^{-1}\right)$ & $242.4_{-1.2}^{+1.0}$ & $\sigma_{\text {jitter }}\left(\mathrm{m} \mathrm{s}^{-1}\right)$ & 25.18 \\
$e$ & $0.036_{-0.014}^{+0.043}$ & $\sqrt{\chi_{v}^{2}}$ & 1.17 \\
$\omega(\mathrm{deg})$ & $240_{-50}^{+50}$ & $\mathrm{rms}\left(\mathrm{m} \mathrm{s}^{-1}\right)$ & 28.3 \\
$m_{2} \sin i\left(M_{\mathrm{J}}\right)$ & $5.4 \pm 0.4$ & $N_{\text {obs }}$ & 35 \\
$a(\mathrm{au})$ & $0.21 \pm 0.01$ & & \\
\hline
\end{tabular}

Notes. $v_{0}$ denotes absolute velocity of the barycenter of the system, offset is a shift in radial velocity measurements between different telescopes, $\sigma_{\text {jitter }}$ is stellar intrinsic jitter as defined in Johnson et al. (2011), RMS is the root mean square of the residuals.

RV bootstrapping method (Murdoch et al. 1993; Kuerster et al. 1997; Marcy et al. 2005; Wright et al. 2007) was employed to assess the uncertainties of the best-fit orbital parameters (see Niedzielski et al. 2015 for more details). The results of the Keplerian analysis are presented in Table 2 and Fig. 1.

\section{Possibility of transits}

The architecture of the system, with the relatively short orbital period of 26.468 days and the semi-major axis of only $7.2 \pm$ 1.0 stellar radii, results in a probability of $13.9 \pm 2.0 \%$ that the planet transits the host star.

The star is expanding because of its evolution toward the giant branch, which means that the planet is expected to be bloated, with the equilibrium temperature $T_{\text {eq }}=1350 \pm 100 \mathrm{~K}$. Assuming that the planetary radius is in a range of between 1 and 2 Jupiter radii, the transits are expected to be $0.3-1.1 \mathrm{mmag}$ deep. The predicted transit duration is between $5.2 \mathrm{~h}$ for grazing transits and $29 \mathrm{~h}$ for central transits. These transit parameters together with possible photometric variability of the host star make transit observations challenging from the ground with telescopes smaller than $2 \mathrm{~m}$. The system could be followed with space-borne instruments such as CHEOPS (Broeg et al. 2013).
The ephemeris of the possible transits is

$T_{\mathrm{t}}(\mathrm{HJD})=2457235.4 \pm 2.6+E \times P$,

where $T_{\mathrm{t}}$ is the mid-transit time for an epoch $E$ (in HJD).

Detecting a transit event would allow determining the planetary radius, orbital inclination, planetary mass, and mean density. The planet would be a very interesting object for studies of planet-star interactions in late stages of stellar evolution. A non-detection would set constraints on the orbital inclination or planetary radius.

\section{Discussion and conclusions}

Given the orbital period of the companion (26.468 days) and the stellar mass $\left(M=1.87 \pm 0.17 M_{\odot}\right)$, stellar radius $(R=$ $\left.6.26 \pm 0.86 R_{\odot}\right)$, and the evolutionary stage of the host $(\log g=$ $3.11 \pm 0.09$ ), the planetary system TYC $3667-1280-1$ is certainly unique. Its host is an evolved A star entering the red giant branch. The planet resides at only 7.2 $\pm 1.0 R_{\star}$ and is on the brink of engulfment, which will occur when its host reaches the radius of $\sim 20 R_{\odot}$ Villaver et al. (2014) in $\sim 1.7 \times 10^{8} \mathrm{yr}$. This fate is only marginally delayed using a different mass-loss prescription (see Villaver et al. 2014).

It is the most compact planetary system ( $a=0.21 \mathrm{au})$ hosted by an evolved $(\log g \leq 3.5)$, intermediate-mass $\left(M_{\star} \geq 1.5 M_{\odot}\right)$ star. The only planetary systems discovered using RV method that bear a resemblance to TYC 3667-1280-1 are HIP $67851 \mathrm{~b}$ (Jones et al. 2015), HD 102956 b (Johnson et al. 2010), and $8 \mathrm{UMib}$ (Lee et al. 2015). However, while the host star of HIP $67851 \mathrm{~b}$ has a similar mass of $1.63 \pm 0.22 M_{\odot}$ and is at the similar stage of stellar evolution $(\log g=3.2 \pm 0.2)$, the planet itself resides on a much wider orbit with $a=0.459 \pm 0.021 \mathrm{au}$ and is much lighter $\left(1.38 \pm 0.15 M_{\mathrm{J}}\right)$. HD $102956 \mathrm{~b}$ is even less massive $\left(0.96 \pm 0.05 M_{\mathrm{J}}\right)$ and is hosted by a less evolved $(\log g=3.5 \pm 0.06), 1.68 \pm 0.11 M_{\odot}$ star, similarly to $8 \mathrm{U} \mathrm{Mi} \mathrm{b,} \mathrm{a}$ $1.5 \pm 0.2 M_{\mathrm{J}}$ planet orbiting a $1.8 \pm 0.1 M_{\odot}, \log g=2.57 \pm 0.03$ star in $0.49 \pm 0.03$ au orbit (Fig. 2).

TYC 3667-1280-1 b is the most massive planet of these, which makes it a strong case for the study of tidal dissipation, similar to WASP-18b (Hellier et al. 2009). The proximity of its evolved luminous host TYC 3667-1280-1 b causes its equilibrium temperature to be very high and places the planet among the hottest Jupiters known (see Fig. 3). This makes it a perfect laboratory for studying inflation processes (Lopez \& Fortney 2016).

The relatively high probability of transits adds to is attractiveness. The estimated probability of $13.9 \pm 2.0 \%$ is high enough to make this worthwhile, even though the expected transit depth is relatively low $(0.3-1.1 \mathrm{mmag})$ and the transit duration may reach up to 29 hours. TYC 3667-1280-1 b certainly deserves more attention and a photometric follow-up in search for transits.

Furthermore, the planetary system of TYC 3667-1280-1 represents an excellent test case on which to study the formation of WJ through Kozai-Lidov oscillations because its mere existence, along with its almost circular orbit, defies the theories of migration through this mechanism. Planets like TYC 3667$1280-1 \mathrm{~b}$ are extremely rare. One object in the complete sample of $\sim 1000$ PTPS stars suggests a frequency of only $\sim 0.1 \%$. However, given one object detected so far within the total PTPS sample of 103 stars with $M_{\star} \geq 1.5 M_{\odot}$, we can estimate that objects like this appear around $\sim 1 \%$ of evolved intermediate-mass stars in our sample. They are apparently as common as HJ around the main-sequence stars. Our discovery suggests that WJ around 


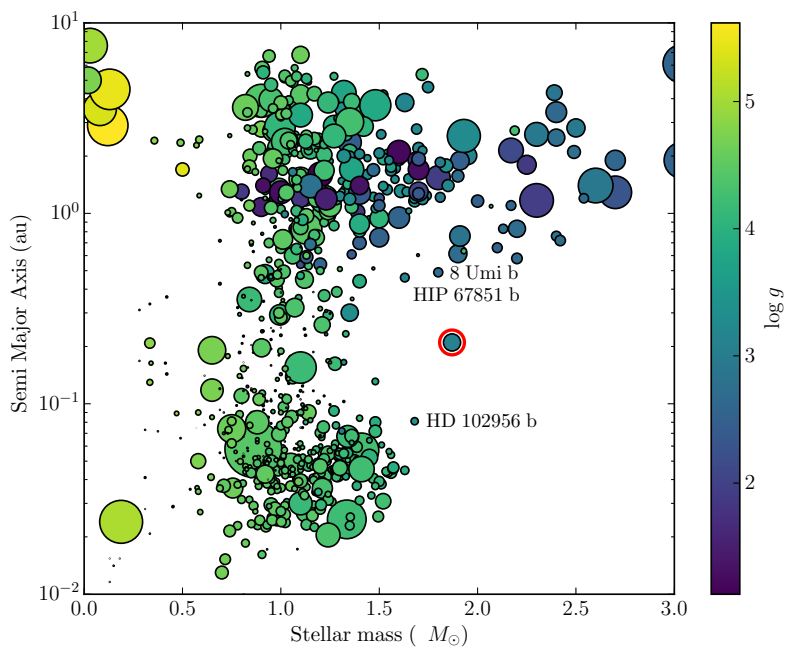

Fig. 2. Orbital period vs. stellar radius plot with the position of TYC 3667-1280-1 b indicated with a red circle. The size of the symbols is proportional to the planetary mass, color indicates $\log g$ of the host star. Data from Exoplanets.eu.

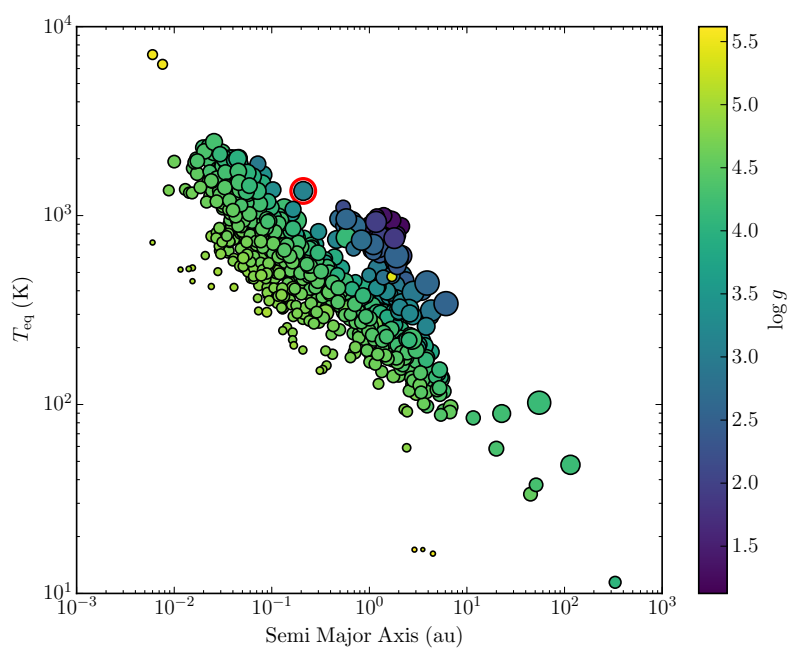

Fig. 3. Planet equilibrium temperature vs. semi-major axis plot with the position of TYC 3667-1280-1 $\mathrm{b}$ indicated with a red circle. The size of the symbols is proportional to the stellar mass, color indicates $\log g$ of the host star. Data from Exoplanets.eu.

intermediate-mass stars survive the evolution of their hosts at the beginning of the red giant branch, where TYC 3667-1280-1 is currently located.

Acknowledgements. We thank the HET and IAC resident astronomers and telescope operators for their support. M.A. acknowledges the Mobility+III fellowship from the Polish Ministry of Science and Higher Education. M.A., A.N., B.D., and MiA were supported by the Polish National Science Centre grant No. UMO-2012/07/B/ST9/04415. E.V. acknowledges support from the Spanish Ministerio de Economía y Competitividad under grant AYA2014-55840P. K.K. was funded in part by the Gordon and Betty Moore Foundation's Data-Driven Discovery Initiative through Grant GBMF4561. This research was supported in part by PL-Grid Infrastructure. The HET is a joint project of the University of Texas at Austin, the Pennsylvania State University, Stanford University, Ludwig- Maximilians-Universität München, and Georg-August-Universität Göttingen. The HET is named in honor of its principal benefactors, William P. Hobby and Robert E. Eberly. The Center for Exoplanets and Habitable Worlds is supported by the Pennsylvania State University, the Eberly College of Science, and the Pennsylvania Space Grant Consortium. This work made use of NumPy (Walt et al. 2011), Matplotlib (Hunter 2007), Pandas (McKinney 2010) and yt (Turk et al. 2011).

\section{References}

Adamczyk, M., Deka-Szymankiewicz, B., \& Niedzielski, A. 2016, A\&A, 587, A119

Adamów, M., Niedzielski, A., Villaver, E., Wolszczan, A., \& Nowak, G. 2014, A\&A, 569, A55

Adamów, M., M., Niedzielski, A., Villaver, E., et al. 2015, A\&A, 581, A94

Baranne, A., Mayor, M., \& Poncet, J. L. 1979, Vist. Astron., 23, 279

Baranne, A., Queloz, D., Mayor, M., et al. 1996, A\&AS, 119, 373

Boley, A. C., Granados Contreras, A. P., \& Gladman, B. 2016, ApJ, 817, L17

Broeg, C., Fortier, A., Ehrenreich, D., et al. 2013, in EPJ Web Conf., 47, 03005

Butler, R. P., Marcy, G. W., Williams, E., et al. 1996, PASP, 108, 500

Charbonneau, P. 1995, ApJS, 101, 309

Cosentino, R., Lovis, C., Pepe, F., et al. 2012, in SPIE Conf. Ser., 8446

Dawson, R. I., \& Chiang, E. 2014, Science, 346, 212

Dawson, R. I., \& Murray-Clay, R. A. 2013, ApJ, 767, L24

Dong, S., Katz, B., \& Socrates, A. 2014, ApJ, 781, L5

Fellgett, P. 1955, Optica Acta, 2, 9

Fogg, M. J., \& Nelson, R. P. 2009, A\&A, 498, 575

Ford, E. B., \& Gregory, P. C. 2007, in Statistical Challenges in Modern Astronomy IV, eds. G. J. Babu, \& E. D. Feigelson, ASP Conf. Ser., 371, 189

Frewen, S. F. N., \& Hansen, B. M. S. 2016, MNRAS, 455, 1538

Goździewski, K., \& Migaszewski, C. 2006, A\&A, 449, 1219

Goździewski, K., Konacki, M., \& Maciejewski, A. J. 2003, ApJ, 594, 1019

Goździewski, K., Maciejewski, A. J., \& Migaszewski, C. 2007, ApJ, 657, 546

Griffin, R. F. 1967, ApJ, 148, 465

Hellier, C., Anderson, D. R., Collier Cameron, A., et al. 2009, Nature, 460, 1098

Høg, E., Fabricius, C., Makarov, V. V., et al. 2000, A\&A, 355, L27

Howard, A. W., Marcy, G. W., Bryson, S. T., et al. 2012, ApJS, 201, 15

Huang, C. X., Wu, Y., \& Triaud, A. H. M. J. 2016, ApJ, submitted [arXiv: 1601.05095]

Hunter, J. D. 2007, Comp. Sci. Eng., 9, 90

Johnson, J. A., Bowler, B. P., Howard, A. W., et al. 2010, ApJ, 721, L153

Johnson, J. A., Clanton, C., Howard, A. W., et al. 2011, ApJS, 197, 26

Jones, M. I., Jenkins, J. S., Rojo, P., Melo, C. H. F., \& Bluhm, P. 2015, A\&A, 573, A3

Kuerster, M., Schmitt, J. H. M. M., Cutispoto, G., \& Dennerl, K. 1997, A\&A, 320,831

Lee, B.-C., Park, M.-G., Lee, S.-M., et al. 2015, A\&A, 584, A79

Lopez, E. D., \& Fortney, J. J. 2016, ApJ, 818, 4

Maciejewski, G., Niedzielski, A., Wolszczan, A., et al. 2013, AJ, 146, 147

Marcy, G. W., \& Butler, R. P. 1992, PASP, 104, 270

Marcy, G. W., Butler, R. P., Vogt, S. S., et al. 2005, ApJ, 619, 570

Markwardt, C. B. 2009, in Astronomical Data Analysis Software and Systems XVIII, eds. D. A. Bohlender, D. Durand, \& P. Dowler, ASP Conf. Ser., 411,251

Mayor, M., \& Queloz, D. 1995, Nature, 378, 355

McKinney, W. 2010, in Proc. 9th Python in Science Conference, eds. S. van der Walt, \& J. Millman, 51

Murdoch, K. A., Hearnshaw, J. B., \& Clark, M. 1993, ApJ, 413, 349

Niedzielski, A., Konacki, M., Wolszczan, A., et al. 2007, ApJ, 669, 1354

Niedzielski, A., Villaver, E., Wolszczan, A., et al. 2015, A\&A, 573, A36

Niedzielski, A., Deka-Szymankiewicz, B., Adamczyk, M., et al. 2016a, A\&A, 585, A73

Niedzielski, A., Villaver, E., Nowak, G., et al. 2016b, A\&A, 588, A62

Nowak, G. 2012, Ph.D. Thesis, Nicolaus Copernicus Univ., Toruń, Poland

Nowak, G., Niedzielski, A., Wolszczan, A., Adamów, M., \& Maciejewski, G. 2013, ApJ, 770, 53

Pepe, F., Mayor, M., Galland, F., et al. 2002, A\&A, 388, 632

Pollacco, D. L., Skillen, I., Collier Cameron, A., et al. 2006, PASP, 118, 1407

Queloz, D. 1995, in New Developments in Array Technology and Applications, eds. A. G. D. Philip, K. Janes, \& A. R. Upgren, IAU Symp., 167, 221

Ramsey, L. W., Adams, M. T., Barnes, T. G., et al. 1998, in SPIE Conf. Ser. 3352, ed. L. M. Stepp, 34

Shetrone, M., Cornell, M. E., Fowler, J. R., et al. 2007, PASP, 119, 556

Tull, R. G. 1998, in SPIE Conf. Ser. 3355, ed. S. D'Odorico, 387

Turk, M. J., Smith, B. D., Oishi, J. S., et al. 2011, ApJS, 192, 9

Villaver, E., Livio, M., Mustill, A. J., \& Siess, L. 2014, ApJ, 794, 3

Walt, S. v. d., Colbert, S. C., \& Varoquaux, G. 2011, Comp. Sci. Engg., 13, 22

Woźniak, P. R., Vestrand, W. T., Akerlof, C. W., et al. 2004, AJ, 127, 2436

Wright, J. T., \& Howard, A. W. 2009, ApJS, 182, 205

Wright, J. T., Marcy, G. W., Fischer, D. A., et al. 2007, ApJ, 657, 533

Wright, J. T., Marcy, G. W., Howard, A. W., et al. 2012, ApJ, 753, 160

Zieliński, P., Niedzielski, A., Wolszczan, A., Adamów, M., \& Nowak, G. 2012, A\&A, 547, A91 


\section{Appendix A: Additional tables}

Table A.1. HET and HRS RV and BS measurements $\left(\mathrm{m} \mathrm{s}^{-1}\right)$ of TYC 3667-1280-1.

\begin{tabular}{lrrrr}
\hline \hline MJD & $\mathrm{RV}$ & $\sigma_{\mathrm{RV}}$ & $\mathrm{BS}$ & $\sigma_{\mathrm{BS}}$ \\
\hline 54394.155075 & 258.5 & 10.3 & -48.4 & 14.4 \\
54398.137124 & 217.8 & 9.3 & -36.9 & 24.0 \\
54748.181551 & -45.9 & 10.5 & -20.8 & 31.5 \\
54779.129988 & -185.2 & 10.0 & -40.3 & 26.9 \\
55184.154144 & 156.9 & 9.8 & -44.6 & 24.1 \\
55469.395590 & -202.0 & 8.9 & -83.8 & 19.7 \\
55500.319306 & 12.1 & 10.6 & 17.5 & 34.0 \\
55895.253142 & -67.2 & 8.8 & -28.3 & 21.9 \\
56127.428623 & -122.8 & 10.6 & -79.8 & 22.8 \\
56232.306667 & -68.9 & 8.7 & -0.4 & 19.4 \\
56280.170359 & 214.9 & 10.2 & -90.6 & 27.1 \\
56295.121470 & 80.6 & 8.7 & -10.7 & 27.9 \\
56314.096829 & -188.1 & 9.2 & -66.5 & 24.8 \\
\hline
\end{tabular}

Table A.2. TNG and HARPS-N RV and BS measurements $\left(\mathrm{m} \mathrm{s}^{-1}\right)$ of TYC 3667-1280-1.

\begin{tabular}{lrrr}
\hline \hline MJD & $\mathrm{RV}$ & $\sigma_{\mathrm{RV}}$ & $\mathrm{BS}$ \\
\hline 56277.0056838 & 5852.76 & 0.52 & -208.5 \\
56293.9512124 & 5497.29 & 0.89 & -83.2 \\
56320.904931 & 5522.61 & 1.44 & -63.3 \\
56560.1855872 & 5609.82 & 1.86 & -150.9 \\
56647.0042254 & 5788.52 & 2.42 & -70.0 \\
56684.8799384 & 5372.04 & 2.27 & 30.2 \\
56895.1655851 & 5412.09 & 2.56 & -98.3 \\
56926.9657616 & 5440.58 & 2.93 & 19.5 \\
56927.2172308 & 5453.96 & 2.99 & 16.2 \\
56969.8405909 & 5640.65 & 1.84 & -137.5 \\
56970.050176 & 5635.57 & 3.56 & -187.8 \\
56970.070991 & 5609.47 & 4.10 & -168.5 \\
56991.9991155 & 5817.72 & 2.75 & -154.6 \\
57034.819799 & 5549.53 & 2.58 & 53.8 \\
57034.9211894 & 5553.18 & 2.77 & 57.9 \\
57034.9663153 & 5555.87 & 2.78 & 65.6 \\
57065.8310694 & 5803.94 & 1.99 & 16.8 \\
57065.8540671 & 5804.62 & 3.24 & 14.9 \\
57196.209713 & 5661.05 & 1.55 & -95.9 \\
57196.2327686 & 5726.66 & 2.09 & 141.2 \\
57238.1151227 & 5426.42 & 1.63 & -103.7 \\
57238.2091972 & 5418.56 & 1.79 & -60.3 \\
\hline
\end{tabular}

